\title{
Recent international trends in psychotropic medication prescriptions for children and adolescents
}

\author{
Hans-Christoph Steinhausen
}

Received: 23 June 2014 / Accepted: 13 October 2014 / Published online: 8 November 2014

(C) Springer-Verlag Berlin Heidelberg 2014

\begin{abstract}
Prescriptions of psychotropic medications have become an important intervention for many children and adolescents with mental disorders, and the rise of these prescriptions is debated intensively both among experts and the public. This review reports some recent international findings on point prevalence rates, cross-country comparisons, and time trends in psychotropic medication prescriptions for children and adolescents. Besides the total prescription rates, figures for antidepressants, antipsychotics, stimulants, and anxiolytics prescriptions are provided. The overall prescription rates of psychotropics in general and the major medication subgroups prescribed to children and adolescents vary substantially between countries with the US consumption being markedly higher than the use in European countries. However, even among the latter there are marked variations in findings. Studies reporting on time trends clearly indicate that there was a marked increase in the use of psychotropics for children and adolescents in the recent past. However, only a single study adjusted prevalence rates for the increasing number of children and adolescents assessed and treated in institutions providing mental health care. Considering the increasing numbers of children and adolescents seen in
\end{abstract}

H.-C. Steinhausen $(\bowtie)$

Research Unit for Child and Adolescent Psychiatry,

Aalborg Psychiatric Hospital, Aalborg University Hospital,

Mølleparkvej 10, 9000 Aalborg, Denmark

e-mail: hc.Steinhausen@kjpd.uzh.ch; hces@rn.dk

\section{H.-C. Steinhausen}

Clinical Psychology and Epidemiology,

Department of Psychology, University of Basel, Basel,

Switzerland

H.-C. Steinhausen

Department of Child and Adolescent Psychiatry,

University of Zurich, Zurich, Switzerland psychiatric services, the increase of psychotropic medications is less strong though still pronounced enough to stimulate further reflections on the use of these interventions.

Keywords Psychopharmacology $\cdot$ Stimulants · Antidepressants · Antipsychotics · Anxiolytics · Children · Adolescents

Key points

Psychotropic medication is an important component of intervention for mental disorders in children and adolescents.

There is a similar general trend in many countries of increasing prescription rates for psychotropic medication in children and adolescents.

Although insufficiently controlled in almost all pharmoco-epidemiological studies, most of this increase is probably due to increasing number of help-seeking patients.

The prescription rates for all major psychotropic medications vary substantially for most countries reflecting both variations in study design and in prescription cultures.

In almost all countries, the prescription rates for stimulants rank highest followed by those for antidepressants and antipsychotics.

\section{Introduction}

Treatment with psychotropic medication has become a cornerstone of intervention for child and adolescent mental disorders. Although the prevalence rates for medication vary considerably between countries, there is the impression both among professionals and the lay public that the prescription rates for these medications are increasing. A recent forum article has put forward the great concern today that children are being over-treated with medication, 
especially in the US [1] and has resulted in various commentaries from international experts including the European perspective $[2,3]$. The present editorial review aims at describing some major trends in the use of psychotropic medications in children and adolescents as reflected in selected major studies published since 2000. No attempt was made to cover all available studies in terms of a systematic study of the entire literature which would go beyond the scope of the present review. Rather, the analytical scheme of the present contribution will look at point prevalence rates in various countries, cross-country comparisons, and time trends in the use of these medications in general and for the major groups of psychotropics, namely, antidepressants, antipsychotics, stimulants, and anxiolytics. Due to very rare reports on the use of mood stabilizers, hypnotics, and alpha-agonists, these medications will not be addressed in the present review.

\section{General use of psychotropics in children and adolescents}

There are a few studies from various countries reporting point prevalence rates in the recent past for various psychotropics combined. For instance, all the hypnotic, anxiolytic, and antidepressant prescriptions in a total population of 120,908 adolescents aged 13-17 years in a southern France area during the year 2002 resulted in a maximum prevalence of $6.3 \%$ for the 17-year-old girls [4]. In a nationwide population-based study covering the total population of 0-17 year olds in Iceland, the overall prevalence of any psychotropic drug use was $4.87 \%$ of children and adolescents in 2007 [5]. Recently reported data from the US National Comorbidity Survey-Adolescent Supplement (2002-2004), a nationally representative survey of 10,123 adolescents aged 13-18 years, indicate that the 12-month prevalence was $7.0 \%$ for the use of at least one psychotropic medication in this age group. Many, albeit far from all adolescents, had mental disorders for which the specific medications are clinically indicated [6].

Several studies performed cross-country comparisons. Psychotropic prescriptions in children and adolescents were compared in the UK and three other European countries with the largest markets for these medications (France, Germany, and Spain), three South American countries with the largest markets for these medications (Argentina, Brazil, and Mexico), and North America (Canada and the USA). It was found that the number of psychotropic prescriptions for children has risen between the years 2000 and 2002 in all nine countries, and seven have shown a significant increase. In this comparative analysis, the UK had the highest percentage increase $(68 \%)$ whereas the lowest was Germany (13\%) [7]. In another comparative study, the annual prevalence of any psychotropic medication in youth for the year 2000 was significantly greater in the US $(6.7 \%)$ than in the Netherlands $(2.9 \%)$ and in Germany $(2.0 \%)[8]$.

In addition, several studies have analyzed general time trends in the use of psychotropic medications in children and adolescents. Changes in the full spectrum of psychotropic medication treatment for youths were analyzed from 1987 to 1996 in a population-based analysis of community treatment data on nearly 900,000 youths enrolled in two US health care systems. The total psychotropic medication prevalence for youths increased two to threefold and included most classes of medication. In this study, the rapid growth since 1991 of Alpha-agonists, neuroleptics, and mood stabilizer anticonvulsants was particularly notable. The 1996 prevalence of any psychotropic medication among youths younger than 20 years was remarkably similar (5.9-6.3 \%) across all three sites. It was concluded that youth psychotropic treatment utilization during the 1990s nearly reached adult utilization rates [9]. In the Netherlands, the year prevalence of antipsychotics, benzodiazepines, antidepressants, and stimulants increased from $1.11 \%$ in 1995 to $2.29 \%$ in 2001 [10].

Prescriptions for children and adolescents over a 15-year period from 1996 to 2010 were analyzed based on the entire dataset of 105,908 patient years in the Danish prescription register covering stimulants, antidepressants, antipsychotics, and anxiolytics. This is the only study that calculated both non-adjusted age-standardized prevalence rates and adjusted age-standardized prevalence rates considering the increase in patient numbers over time. There was a ninefold increase from a low of 1.34 per 1,000 person-years in 1996 to a high of 12.35 per 1,000 person-years in 2010 in total dispensed psychotropic medication for children and adolescents in Denmark which was only twofold after adjusting for increasing patient numbers [11].

\section{Antidepressants}

Whereas there are quite a number of recent time-trend reports on the changing use of antidepressants in children and adolescents, there are only a few studies restricting their focus on point-prevalence studies in various countries. In an Italian region, prescriptions of antidepressants in 2001 yielded a prevalence of 2.78 subjects per 1,000 inhabitants during 12 months. The prevalence progressively rose with age in both sexes and a female/male difference was evident in adolescents [12]. The US National Comorbidity Survey-Adolescent Supplement (2002-2004)—reported that $3.9 \%$ of adolescents aged 13-18 years had received antidepressants during a 12-month period. In this study, the antidepressants were the most commonly used 
psychotropic medication [6]. In the Icelandic investigation, 23.4 per 1,000 children and adolescents in 2007 received antidepressants so that antidepressants were the second most prevalent psychotropic medication group [5].

One study compared antidepressant prevalence data in youths across three western European countries (Denmark, Germany, and the Netherlands) with US regional data. The population-based analysis was based on administrative claims data for the year 2000 in 0 to 19 -year-old enrollees who were part of the insured populations from these four countries having a total of from 72,570 to 480,680 members. Utilization of antidepressants in the US dataset $(1.63 \%)$ exceeded that of three Western European countries (prevalence ranged from 0.11 to $0.54 \%$ ) by at least threefold. In addition, there were major variations in the use of subclasses; tricyclic antidepressants (TCAs) predominated in Germany while selective serotonin reuptake inhibitors (SSRIs) predominated in the US, Denmark, and the Netherlands [13].

Several studies have analyzed time trends of antidepressant prescription data in various countries. Prescriptions for children and adolescents were analyzed in the UK based on the General Practise Research Database between 1992 and 2001. Among the prescriptions, $55.7 \%$ were for TCAs, $41.3 \%$ for SSRIs, and $2.9 \%$ for other antidepressants. The antidepressant prevalence increased 1.7-fold during the observation period with TCAs prevalence decreasing by $30 \%$ from 3.6 to 2.5 per 1,000 and SSRIs prevalence increasing 10 times from 0.5 to 4.6 per 1,000 . It was concluded that SSRIs had gained popularity for the treatment of depression compared with TCAs [14].

In the US, the Medical Expenditure Panel Survey (MEPS) database for the years 1997-2002 was analyzed. The MEPS is a yearly survey of a nationally representative sample of civilian, non institutionalized US Households. The percentage of users increased from $1.3 \%$ in 1997 to $1.8 \%$ in 2002 with adolescent use $(2.1 \%$ in 1997 versus $3.9 \%$ in 2002) accounting for the increase whereas there was no change among children younger than 13 years. Also among adolescents, the use rate remained stable during the years from 2000 to 2002. The increase was caused by the use of SSRIs and other newer antidepressants, whereas the use of TCAs remained stable in adolescents and declined in prepubertal children. The use of antidepressant was similar among males and females [15]. Another recent US study compared the MEPS data from 1996 to 2005 and found that the rate of antidepressant treatment increased from $5.84 \%$ in 1996 to $10.12 \%$ in 2005 indicating a broad marked increase in antidepressant treatment [16].

Similar to the observations in the US, the prevalence of antidepressants prescribed in Germany remained rather stable in more recent years, namely, at 3.43 per 1,000 in 2000 and 3.74 per 1,000 in 2003. At this time, St John's Wort products, which are approved for antidepressant use by the German drug agency, and TCAs accounted for more than $80 \%$ of antidepressant use and SSRIs represented only $15 \%$ of antidepressant use. However, there was a doubling of the use of SSRIs over the 4-year period. Adolescent girls aged 15-19 years had utilization rates ranging from 11.44 to 13.82 per 1,000 , and accounted for most antidepressant use. Overall, females were twice as likely as males to get an antidepressant [17].

Limitations in licensing of antidepressants in Germany excluding the use of SSRIs except fluoxetine since 2006 have led to frequent off-label prescriptions. A recent study characterised antidepressant medication use with a particular focus on off-label prescribing in a retrospective cohort study including cross-sectional analyses based on claims data of 2,599,685 patients up to 17 years of age. The prevalence of antidepressant medication use was 1.84 per 1,000 children in 2004, 1.57 per 1,000 in 2005, and 1.66 per 1,000 in 2006. Of all prescribed antidepressants $42.09 \%$ were TCAs, $34.58 \%$ were SSRIs, $16.47 \%$ were St John's wort preparations, and $6.86 \%$ were other antidepressants [18].

The recent Danish analysis of prescriptions of psychotropics over the 15-year period from 1996 to 2010 based on the national prescription register observed an increase from 0.28 to 2.67 per 1,000 person-years. However, after adjusting for the increasing base rate of patients over time, the prevalence rates were only less than two times higher at the end compared to the beginning of the observation period. As in other studies, the increase in prescription rates for antidepressants was mainly due to increased use among the 14-17 year olds [11].

\section{Antipsychotics}

Only a few studies reported point prevalence rates of the use of antipsychotic medication in the recent past. Based on a questionnaire sent out to all child and adolescent psychiatry departments and consultants with a 6 or $63 \%$ response rates, respectively, $6.4 \%$ of treated children and adolescents in Denmark on October, 1 in 2002 received antipsychotic medication. Two-thirds of the patients had been treated with a second-generation antipsychotic (SGA) [19]. In the above mentioned US National Comorbidity Survey-Adolescent Supplement (2002-2004)—during a 1 -year period $0.5 \%$ of the adolescents had received antipsychotics [6].

Cross-country comparisons indicate that the use of antipsychotics was 1.5-2.2 times higher in the US than in the Netherlands and Germany in 2000 with the atypical antipsychotic subclass representing only $5 \%$ of antipsychotic 
use in Germany, but $48 \%$ in the Netherlands and $66 \%$ in the US [8].

Various studies reported time trends in the use of antipsychotic medication. In the UK, the overall prevalence of use of all antipsychotics increased from 0.39 users per 1,000 in 1992 to 0.77 users per 1,000 in 2005 [20]. Between 1993 and 2002, a sharp increase in antipsychotic treatment has also been observed among children and adolescents in office-based medical practice in the US. From 2000 to 2002, $9.2 \%$ of all mental health visits and $18.3 \%$ of visits to psychiatrists included antipsychotic treatment and $92.3 \%$ of visits with prescription of an antipsychotic included a SGA medication [21]. In Canada, antipsychotic drug recommendations for children with ADHD more than tripled between 2005 and 2009 . The majority of antipsychotic drug recommendations were for SGA with attention-deficit/hyperactivity disorder (ADHD) being the most common therapeutic indication associated with an SGA recommendation [22]. In Texas, from 1996 to 2000, an additional 12.25 children and adolescents per 1,000 enrolees $(+160 \%)$ were prescribed antipsychotics [23].

The above-mentioned recent analysis of Danish prescriptions of antipsychotic medication over 15 years found an increase from 0.31 per 1,000 person-years in 1996 to 2.05 per 1,000 person-years in 2010 . Antipsychotics were significantly more frequently given to males and the 1417 year olds in each year [11].

\section{Stimulants}

There is evidence from various studies that among the various psychotropic drugs the stimulants are most widely prescribed in quite a number of countries including the US [9], the Netherlands [10], Iceland [5], and Denmark [11]. The 2007 prevalence of attention-deficit hyperactivity disorder (ADHD) drug use including MPH as the most frequently prescribed drug among the total Nordic population varied from 1.23 per 1,000 inhabitants in Finland to 12.46 per 1,000 in Iceland [24].

Further studies have documented the increasing prescription rates for various countries. Data collected in two Medicaid programs and one health maintaining organization for three one year periods in 1991, 1993, and 1995 showed that there has been a 1.7- to 3.1-fold increase of prescriptions for preschoolers between 1991 and 1995 in the US [25]. Other data from the US show that an estimated 35 per 1,000 of US children received stimulant medication in 2008, up from 24 per 1,000 in 1996 [26]. The most recent US report based on the 2011 National Survey of Children's Health (NSCH) used parent reported data and found that the prevalence of medicated ADHD increased by $28 \%$ from 2007 to 2011 [27]. In the Netherlands, the prevalence of stimulants increased from 1.5 per 1,000 in 1995 to 7.4 per 1,000 in 1999 [28]. In France, the annual prevalence of methylphenidate per 1,000 was 1.1 in 2003, 1.5 in 2004, and 1.8 in 2005 [29]. A German cross-sectional study analyzed data from a random sample of some 50,000-63,000 children and adolescents per calendar year who were insurants of a major insurance company in one federal state for the years 2000-2007. The study found that parallel to the increasing prevalence rates of ADHD the prescription rates for methlphenidate almost doubled from 0.54 to $1.06 \%$ for school-aged children [30].

A recent study from Sweden covered the total population cohort of all individuals aged 6-45 years, alive during any calendar year from 2006 to 2009. In this cohort, other medications used for ADHD treatment, namely, atomoxetine, amphetamine, or dexamphetamine were also considered in the analysis. The dispensing prevalence increased from 2.93 per 1,000 in 2006 to 6.98 per 1,000 in 2009 . The increase was largest for adults and greater in women than in men [31].

In the above mentioned large Danish study based on a nationwide dataset covering all prescriptions over the 15-year period between 1996 and 2010, the prevalence rates increased from 0.31 per 1,000 person-years in 1996 to 7.29 per 1,000 person-years in 2010. After adjusting for the increasing overall number of patients, there was an eightfold increase of stimulant prescriptions over time with males receiving significantly more stimulants than females. Furthermore, the increase was significant for all age groups from 0 to 17 years [11].

Another recent Danish register study used data on all children born between 1990 and 2001 and found that from 2003 to 2010 youth 6-13 years of age with autism spectrum disorders, ADHD, and other psychiatric disorders had 4.7, 6.3 and 5.5-fold increases, respectively, in prescription rates of ADHD medications which besides methylphenidate also included the less frequent prescriptions for dexamphetamine and atomoxetine [32].

\section{Anxiolytics}

Few recent studies have reported on point prevalence rates of medications using anxiolytics in children and adolescents. In the analysis of prescriptions, in 2002 for 13-17 year olds in a southern France area, 20.1 per 1,000 received anxiolytics [4]. The US National Comorbidity Survey_Adolescent Supplement (2002-2004)—found a prevalence rate of $0.8 \%$ for anxiolytics in 13-18 year olds [6]. Although rarely used, anxiolytics were twice as common in Dutch $(0.73 \%)$ as in US $(0.49 \%)$ and German $(0.41 \%)$ youth [8]. 
Two studies have reported rather stable time trend in the use of anxiolytics in children and adolescents. In Iceland, the prevalence ranged between 1.5 and 2.0 per 1,000 in the 2003-2007 period [5], and in the nationwide Danish prescription register study, the prevalence rates were 0.44 per 1,000 person-years in 1996 and 0.33 per 1,000 personyears in 2010 indicating no significant change over time. No significant sex differences in the prescription rates were found but the 14-17 year olds had the highest rate [11].

\section{Comment}

The present review of the use of various psychotropics in children and adolescents centred around three major issues, namely, point prevalence rates in various countries, crossnational comparisons of these rates, and time trends. A synthesis of the various study findings is impeded by pronounced differences in study designs, databases, and analytical procedures. Furthermore, the variability in the use of medications reflects differences in diagnostic systems, the availability and use of clinical practice guidelines, drug regulation, health services organization, availability and allocation of financial resources, and cultural attitudes toward childhood behavioral and emotional disturbances [33]. In addition to these limitations, the interpretation of the findings of the few studies that performed direct cross-national comparisons of prevalence rates of prescribed psychotropic medication is hampered by lacking representativeness of the samples, rather short time periods of data collection in the majority of studies and varying composition of medications under study.

Even with these considerations in mind, one has to conclude that the findings on point prevalence rates indicate marked international differences in the frequencies of prescription rates across countries both in the total amount of medications and for the various major psychotropic medication groups. Overall, the use of all psychotropics is many times greater in the US than in all other countries. However, also in Europe there is a marked gradient of prescription rates with, for instance, Icelandic children and adolescents receiving higher prescription rates than those in other Scandinavian countries and with Danish children and adolescents having rather low prescription rates. A detailed comparison of the various point prevalence rates for the total medications and the various subgroups between the Danish and other international findings has been provided in a recent publication [11].

Central to the present review of recent reports on the epidemiology of psychotropic medication use in children and adolescents is the finding that there are similar international trends of an increasing use of psychotropic medications in children and adolescents in the recent past. However, these trends differ markedly in magnitude reflecting a variety of factors of influence [33]. So far, sampling effects have not been considered sufficiently. The majority of studies are based on surveys or local samples from a circumscribed area with often unknown representativeness. Accordingly, data in these studies came from audits drawn from medical practitioners in various international regions [7] or from other regional sources [12, 13], insurance companies [8, 13, 17], Medicaid in various US states $[9,13,23]$, regional retail pharmacies in the Netherlands [10, 13, 28], the national health insurance fund for self-employed workers in France [29], or panels of a relatively small number of general practitioners in the UK [20].

Greater success in gaining representativeness may have been achieved in studies based on representative household panels like the representative annual survey of US Households $[15,26]$. A complete coverage of nationwide populations of prescriptions has only been received in the two recent Danish register studies based on either complete birth cohorts [32] or the complete population of children and adolescents receiving psychotropic medications [11].

Furthermore, interpretations of the magnitude of time trends are hampered by the fact that the majority of longitudinal studies were based on rarely more than two observation points in time. In addition, none of the study except the Danish register study on time trends [11] controlled for changing total base-rates of number of patients over the years. Thus, these studies do not sufficiently take into account that much of the increase of prescriptions is due to an increasing awareness for mental problems in children and adolescents and higher referral rates to specialized institutions and professionals in the recent past letting alone the question whether or not the true prevalence rates of mental disorders in this age range might even have increased per se.

Overall, in the recent past, the use of psychotropics in children and adolescents has increased remarkably, and the gap between adult and youth medication rates for mental disorders has narrowed substantially. As Rapoport concluded recently, the use of new treatments, such as stimulants and SSRIs, not only brought help to otherwise treatment-refractory patients but also has contributed to an over-acceptance of reductionistic biology [1]. Furthermore, market forces with the pharmaceutical companies as powerful actors in health care delivery systems also drove the process of over-prescribing with a large proportion of prescriptions without proper clinical indication. However, as Taylor has pointed out recently, considering the large group of insufficiently diagnosed or undetected problems of mental health in children and adolescents, undertreatment is perhaps a bigger problem globally than overmedication [3]. 
Conflict of interest H.-C. Steinhausen has worked in the past as an advisor and speaker for the following pharmaceutical companies: Janssen-Cilag, Eli Lilly, Novartis, Medice, Shire, and UCB. More than three years ago, he has also received unrestricted grants for postgraduate training courses or conferences and research by JanssenCilag, Eli Lilly, Novartis, Medice, and Swedish Orphan International.

\section{References}

1. Rapoport JL (2013) Pediatric psychopharmacology: too much or too little? World Psychiatry 12:118-123

2. Steinhausen HC (2013) A European perspective on paedo-psychiatric pharmacoepidemiology. World Psychiatry 12:131-132

3. Taylor E (2013) Pediatric psychopharmacology: too much and too little. World Psychiatry 12:124-125

4. Mancini J, Thirion X, Masut A, Saillard C, Pradel V, Romain F, Pastor MJ, Coudert C, Micallef J (2006) Anxiolytics, hypnotics, and antidepressants dispensed to adolescents in a French region in 2002. Pharmacoepidemiol Drug Saf 15:494-503

5. Zoega $\mathrm{H}$, Baldursson $\mathrm{G}$, Hrafnkelsson $\mathrm{B}$, Almarsdottir $\mathrm{AB}$, Valdimarsdottir U, Halldorsson M (2009) Psychotropic drug use among Icelandic children: a nationwide population-based study. J Child Adolesc Psychopharmacol 19:757-764

6. Olfson M, He JP, Merikangas KR (2013) Psychotropic medication treatment of adolescents: results from the national comorbidity survey-adolescent supplement. J Am Acad Child Adolesc Psychiatry 52:378-388

7. Wong IC, Murray ML, Camilleri-Novak D, Stephens P (2004) Increased prescribing trends of paediatric psychotropic medications. Arch Dis Child 89:1131-1132

8. Zito JM, Safer DJ, de Jong-van den Berg LT, Janhsen K, Fegert JM, Gardner JF, Glaeske G, Valluri SC (2008) A three-country comparison of psychotropic medication prevalence in youth. Child Adolesc Psychiatry Ment Health 2:26 1753-2000-2-26

9. Zito JM, Safer DJ, DosReis S, Gardner JF, Magder L, Soeken K, Boles M, Lynch F, Riddle MA (2003) Psychotropic practice patterns for youth: a 10-year perspective. Arch Pediatr Adolesc Med 157:17-25

10. Hugtenburg JG, Heerdink ER, Egberts AC (2004) Increased psychotropic drug consumption by children in the Netherlands during 1995-2001 is caused by increased use of methylphenidate by boys. Eur J Clin Pharmacol 60:377-379

11. Steinhausen HC, Bisgaard C (2014) Nationwide time trends in dispensed prescriptions of psychotropic medication for children and adolescents in Denmark. Acta Psychiatr Scand 129:221-231

12. Percudani M, Barbui C, Fortino I, Petrovich L (2005) Worrying patterns of out-patient psychotropic drug prescribing in children and adolescents. Psychother Psychosom 74:189-190

13. Zito JM, Tobi H, de Jong-van den Berg LT, Fegert JM, Safer DJ, Janhsen K, Hansen DG, Gardner JF, Glaeske G (2006) Antidepressant prevalence for youths: a multi-national comparison. Pharmacoepidemiol Drug Saf 15:793-798

14. Murray ML, de Vries CS, Wong IC (2004) A drug utilisation study of antidepressants in children and adolescents using the General Practice Research Database. Arch Dis Child 89:1098-1102

15. Vitiello B, Zuvekas SH, Norquist GS (2006) National estimates of antidepressant medication use among US children, 19972002. J Am Acad Child Adolesc Psychiatry 45:271-279

16. Olfson M, Marcus SC (2009) National patterns in antidepressant medication treatment. Arch Gen Psychiatry 66:848-856

17. Fegert JM, Kolch M, Zito JM, Glaeske G, Janhsen K (2006) Antidepressant use in children and adolescents in Germany. J Child Adolesc Psychopharmacol 16:197-206
18. Dorks M, Langner I, Dittmann U, Timmer A, Garbe E (2013) Antidepressant drug use and off-label prescribing in children and adolescents in Germany: results from a large population-based cohort study. Eur Child Adolesc Psychiatry 22:511-518

19. Deurell M, Weischer M, Pagsberg AK, Labianca J (2008) The use of antipsychotic medication in child and adolescent psychiatric treatment in Denmark. A cross-sectional survey. Nord J Psychiatry $62: 472-480$

20. Rani F, Murray ML, Byrne PJ, Wong IC (2008) Epidemiologic features of antipsychotic prescribing to children and adolescents in primary care in the United Kingdom. Pediatrics 121:1002-1009

21. Olfson M, Blanco C, Liu L, Moreno C, Laje G (2006) National trends in the outpatient treatment of children and adolescents with antipsychotic drugs. Arch Gen Psychiatry 63:679-685

22. Pringsheim T, Lam D, Patten SB (2011) The pharmacoepidemiology of antipsychotic medications for Canadian children and adolescents: 2005-2009. J Child Adolesc Psychopharmacol 21:537-543

23. Patel NC, Sanchez RJ, Johnsrud MT, Crismon ML (2002) Trends in antipsychotic use in a Texas medicaid population of children and adolescents: 1996 to 2000. J Child Adolesc Psychopharmacol $12: 221-229$

24. Zoega H, Furu K, Halldorsson M, Thomsen PH, Sourander A, Martikainen JE (2011) Use of ADHD drugs in the Nordic countries: a population-based comparison study. Acta Psychiatr Scand 123:360-367

25. Zito JM, Safer DJ, DosReis S, Gardner JF, Boles M, Lynch F (2000) Trends in the prescribing of psychotropic medications to preschoolers. JAMA 283:1025-1030

26. Zuvekas SH, Vitiello B (2012) Stimulant medication use in children: a 12-year perspective. Am J Psychiatry 169:160-166

27. Visser SN, Danielson ML, Bitsko RH, Holbrook JR, Kogan MD, Ghandour RM, Perou R, Blumberg SJ (2014) Trends in the parent-report of health care provider-diagnosed and medicated attention-deficit/hyperactivity disorder: United States, 2003-2011. J Am Acad Child Adolesc Psychiatry 53:34-46

28. Schirm E, Tobi H, Zito JM, de Jong-van den Berg LT (2001) Psychotropic medication in children: a study from the Netherlands. Pediatrics 108:E25

29. Knellwolf AL, Deligne J, Chiarotti F, Auleley GR, Palmieri S, Boisgard CB, Panei P, Autret-Leca E (2008) Prevalence and patterns of methylphenidate use in French children and adolescents. Eur J Clin Pharmacol 64:311-317

30. Schubert I, Koster I, Lehmkuhl G (2010) The changing prevalence of attention-deficit/hyperactivity disorder and methylphenidate prescriptions: a study of data from a random sample of insurees of the AOK Health Insurance Company in the German State of Hesse, 2000-2007. Dtsch Arztebl Int 107:615-621

31. Zetterqvist J, Asherson P, Halldner L, Langstrom N, Larsson H (2013) Stimulant and non-stimulant attention deficit/hyperactivity disorder drug use: total population study of trends and discontinuation patterns 2006-2009. Acta Psychiatr Scand 128:70-77

32. Dalsgaard S, Nielsen HS, Simonsen M (2013) Five-fold increase in national prevalence rates of attention-deficit/hyperactivity disorder medications for children and adolescents with autism spectrum disorder, attention-deficit/hyperactivity disorder, and other Psychiatric disorders: a Danish register-based study. J Child Adolesc Psychopharmacol 23:432-439

33. Vitiello B (2008) An international perspective on pediatric psychopharmacology. Int Rev Psychiatry 20:121-126 